\title{
Iterative Turbo Channel Estimation for OFDM System over Rapid Dispersive Fading Channel
}

\author{
Ming Zhao, Student Member, IEEE, Zhenning Shi, Member, IEEE, and Mark C. Reed, Senior Member, IEEE
}

\begin{abstract}
Coherent OFDM detection requires accurate channel state information (CSI). Mobile radio channels are both time and frequency dispersive, especially at high vehicular speeds, which makes channel estimation a challenging problem in system design. Conventional preamble-based and pilot-aided channel estimation require numerous reference signals, which significantly compromises the system throughput. This paper proposes a novel low complexity iterative turbo channel estimation technique which makes use of preamble, pilots and soft decoded data information in an iterative fashion to improve the system performance over the time and frequency selective fading channel while maintaining the system throughput. The numerical and analytical results show that the proposed technique can approach the performance of systems with perfect CSI with much fewer preamble and pilots symbols compared to existing channel estimation methods.
\end{abstract}

Index Terms-OFDM, channel estimation, turbo iterative, rapid dispersive fading channel.

\section{INTRODUCTION}

$\mathbf{O}$ RTHOGONAL Frequency Division Multiplexing (OFDM) is an attractive technique for high data rate transmission over wireless channels. The most important advantage of an OFDM system over a single carrier system is that it transforms the frequency selective channel into a parallel collection of flat fading subchannels, which simplifies the equalization at the receiver. OFDM has been adopted in several wireless standards such as digital audio broadcasting (DAB), digital video broadcasting (DVB-T), the IEEE 802.11a [1] Wireless Local Area Network (WLAN) standard and the IEEE 802.16a/e [2], [3] Metropolitan area network (MAN) standard. OFDM is also a potential candidate of next generation $(4 \mathrm{G})$ mobile wireless communications.

With the knowledge of channel state information (CSI), coherent detection can be performed on OFDM symbols. Realistic mobile radio channels are characterized by the time and frequency dispersive nature due to the multipath delay profile and the Doppler spread of the channel. Fig.1 shows the channel frequency response of IMT-2000 vehicular-A channel [4] over subcarriers and OFDM symbols at vehicular speeds

Manuscript received February 28, 2007; revised June 7, 2007, August 23, 2007, and October 15, 2007; accepted October 17, 2007. The associate editor coordinating the review of this paper and approving it for publication was X.-G. Xia. This work was supported by National ICT Australia, and the Research School of Information Science and Engineering, Australian National University.

M. Zhao is with the Research School of Information Science and Engineering, Australian National University, and National ICT Australia (e-mail: Ming.zhao@rsise.anu.edu.au).

Z. Shi and M. C. Reed are with National ICT Australia (e-mail: \{Zhenning.shi, Mark.reed\}@ nicta.com.au).

Digital Object Identifier 10.1109/TWC.2008.070228.
$3 \mathrm{kmh}, 120 \mathrm{kmh}$, and $333 \mathrm{kmh}$ respectively for the carrier frequency of $5 \mathrm{GHz}$. It can be seen that the channel variation becomes more significant in both time and frequency domain as mobility increases. Therefore, rapid dispersive fading channel with time and frequency selectivity makes channel estimation and tracking a challenging problem in OFDM system design.

In order to estimate selective channels, training signals are employed in OFDM packets. In the uplink, pilot signals are inserted in OFDM data symbols, while a preamble is transmitted prior to the data symbols in the the downlink OFDM frames. More specifically, conventional OFDM systems [1]-[3] assume the channel is static within one frame, and only use channel estimates obtained from the preamble for data symbol detection. Such approach performs well in static channels but incurs a severe performance degradation in the rapid dispersive fading channel. In [5], Dowler et al. proposed a data derived method, which uses decoded hard decision data of the current symbol to re-estimate the channel for the next symbol. This method partially tracks the channel variation, but also introduces delays and error propagation.

In the uplink transmission, pilots are often multiplexed into the data sequence and channel estimation can be performed by interpolation. Negi et al. [6] proposed least square (LS) based channel estimation and discussed optimal pilot spacing. In [7]-[9], channel estimators for OFDM system have been proposed based on the singular value decomposition (SVD) and frequency domain filtering. Time domain filtering has been proposed in [10] to further improve the channel estimator. Extended from Beek's work, Li et al. investigated the correlation of channel frequency response over times and frequency and proposed a robust minimum mean-squareerror (MMSE) channel estimator [11]. On the other hand, complexity prohibits their application to practical systems.

In order to resolve time selective channels, Stamoulis et al. [12] developed a channel estimator based on linear interpolation of partial channel information. Shin et al. [13] approximated LMMSE estimation by representing the channel in basis expansion model (BEM) to obtain the channel impulse response from interpolation of the partial channel information with discrete orthogonal legendre polynomials. Schniter [14], [15] proposed channel estimation using FFT and specific timedomain pilot signals, however, due to the utilization of timedomain pilot signals, it may not be compatible with existing OFDM standards. Furthermore, although above methods can track the rapid dispersive channel, system throughput is sacrificed due to the enormous amount of pilots inserted.

Emerging iterative receivers based on the Turbo principle [16] are shown to be able to provide near-optimal performance 


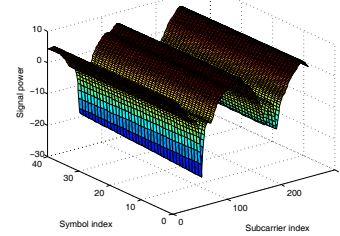

(a) $3 \mathrm{kmh}$

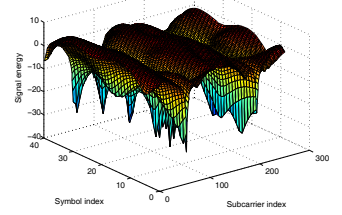

(b) $120 \mathrm{kmh}$

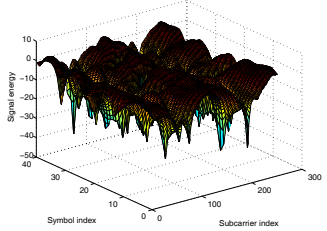

(c) $333 \mathrm{kmh}$

Fig. 1. Time and frequency selective channel with carrier frequency $5 \mathrm{GHz}$.

in space-time processed systems with ideal CSI. When the CSI is not available, Li et al. [17]-[19] proposed decisiondirected LS channel estimator and its simplified variant for space-time trellis-coded (STTC) OFDM systems. Song et al. [20] proposed iterative joint zero forcing (ZF) channel estimation and signal detection algorithm based on hard decision feedback. Wang et al. [21], [22] proposed an iterative receiver for space-time block-coded (STBC) OFDM system based on expectation-maximization (EM) approach. Moon et al. [23] developed an iterative receiver well suited for wireless local area network (WLAN). However, under high mobility situation, aforementioned receivers have degraded performance.

To address dispersive channels, Park et al. [24] proposed an iterative channel estimator by employing time and frequency domain MMSE filters for mobile radio channels. Tomasin et al. [25] proposed an iterative receiver with ICI cancellation and MMSE channel estimation for extremely high mobility condition. These receivers are computationally complex and infeasible for practical systems.

In this paper, a low complexity iterative channel estimation technique that uses a turbo processing approach [16] is proposed. Compared to conventional non-iterative channel estimation methods, the proposed technique makes use of preamble, pilots and soft decoded data information to track the channel frequency response in every OFDM symbol within the data packet or data frame. Compared to existing iterative channel estimation methods, a three-stage estimation scheme is proposed to reduce the complexity and adapt the channel estimates w.r.t the feedback information. More precisely, we estimate the channel based on the improving a priori information of the decoded data, preamble and the pilots by adaptively weighting the statistics according to the respective levels of reliability. The performance of channel estimation is significantly enhanced which in turn leads to improved system performance. The proposed technique also allows for high throughput transmission since there is a substantial saving on the number of preambles and pilots required.

The rest of the paper is organized as follows. In Section II, the OFDM system under time and frequency selective fading channel is described. Section III briefly introduces the iterative receiver. In Section IV, the novel iterative turbo channel estimation technique is proposed. In Section V, lower bounds in terms of MSE for two advanced methods are derived for MLE and MMSE channel estimators. This is used as the benchmark for performance comparison. Section VI discusses the complexity of the proposed channel estimator. Simulation results are shown in Section VII. Section VIII concludes the paper. Matrices and vectors are denoted by symbols in bold face and $(\cdot)^{*},(\cdot)^{T}$ and $(\cdot)^{H}$ represent complex conjugate, transpose and Hermitian transpose. $E\{\cdot\}$ denotes the statistical expectation. $[\mathbf{X}]_{i, j}$ indicates the $(i, j)$ th elements of a matrix $\mathbf{X}$, and similarly, $[\mathbf{x}]_{i}$ indicates the element $i$ in a vector $\mathbf{x}$. Finally, $\{x\}$ represents the sequence $x$.

\section{SyStem MOdEL}

The discrete-time OFDM system with $N$ subcarriers considered in this paper is shown in Fig. 2. The information bits $\left\{\mathbf{b}^{(i)}\right\}$ are first encoded into coded bits $\left\{\mathbf{d}^{(i)}\right\}$, where $i$ is the time index. These coded bits are interleaved into a new sequence of $\left\{\mathbf{c}^{(i)}\right\}$, mapped into $M$-ary complex symbols and serial-to-parallel (S/P) converted to a data sequence of $\left\{\mathbf{X}_{d}^{(i)}\right\}$. Pilot sequence $\left\{\mathbf{X}_{P}^{(i)}\right\}$ are inserted into data sequence $\left\{\mathbf{X}_{d}^{(i)}\right\}$ at pilot subcarriers to form an OFDM symbol of $N$ frequency domain signals represented as vector $\mathbf{X}^{(i)}=\left[X^{(i)}(0), X^{(i)}(1), \cdots, X^{(i)}(N-1)\right]^{T} . N$-point IDFT is performed on $\mathbf{X}^{(i)}$, which is given by:

$$
x^{(i)}(n)=\frac{1}{\sqrt{N}} \sum_{k=0}^{N-1} X^{(i)}(k) \cdot \exp \frac{j 2 \pi k n}{N},
$$

where $0 \leq n \leq N-1$. After adding the $\mathrm{CP}$ of length $G$, the OFDM symbol is converted into a time domain sample vector $\mathbf{x}^{(\mathbf{i})}=\left[x^{(i)}(-G), x^{(i)}(-G+1), \cdots, x^{(i)}(N-1)\right]^{T}$. These time domain samples are then digital to analog converted and transmitted over the multipath fading channel.

The multipath fading channel can be modeled by the timevariant discrete impulse response with $h^{(i)}(n, l)$ representing the fading coefficient of the $l^{t h}$ path at time $n$ for $i^{\text {th }}$ OFDM symbol. The fading coefficients are modeled as zero mean complex Gaussian random variables. Based on the wide sense stationary uncorrelated scattering (WSSUS) assumption, the fading coefficients for different paths are statistically independent, while the fading coefficients for a particular path are correlated over time. The autocorrelation function of $h^{(i)}(n, l)$ is given by [26]:

$$
E\left\{h^{(i)}(n, l) \cdot h^{(i)}(m, l)^{*}\right\}=\alpha_{l} \cdot J_{0}\left(2 \pi(n-m) f_{m} T_{s}\right),
$$

where $J_{0}(\cdot)$ is the first kind of Bessel function of zero order, $T_{s}=1 / B W$ is the sample time, $B W$ is the bandwidth of OFDM system, $f_{m}$ is the maximum Doppler spread and $\alpha_{l}$ is the average power of the $l^{t h}$ path. The channel gain is normalized as given by:

$$
\sum_{l=0}^{L-1} E\left\{\left\|h^{(i)}(n, l)\right\|^{2}\right\}=\sum_{l=0}^{L-1} \alpha_{l}=1,
$$




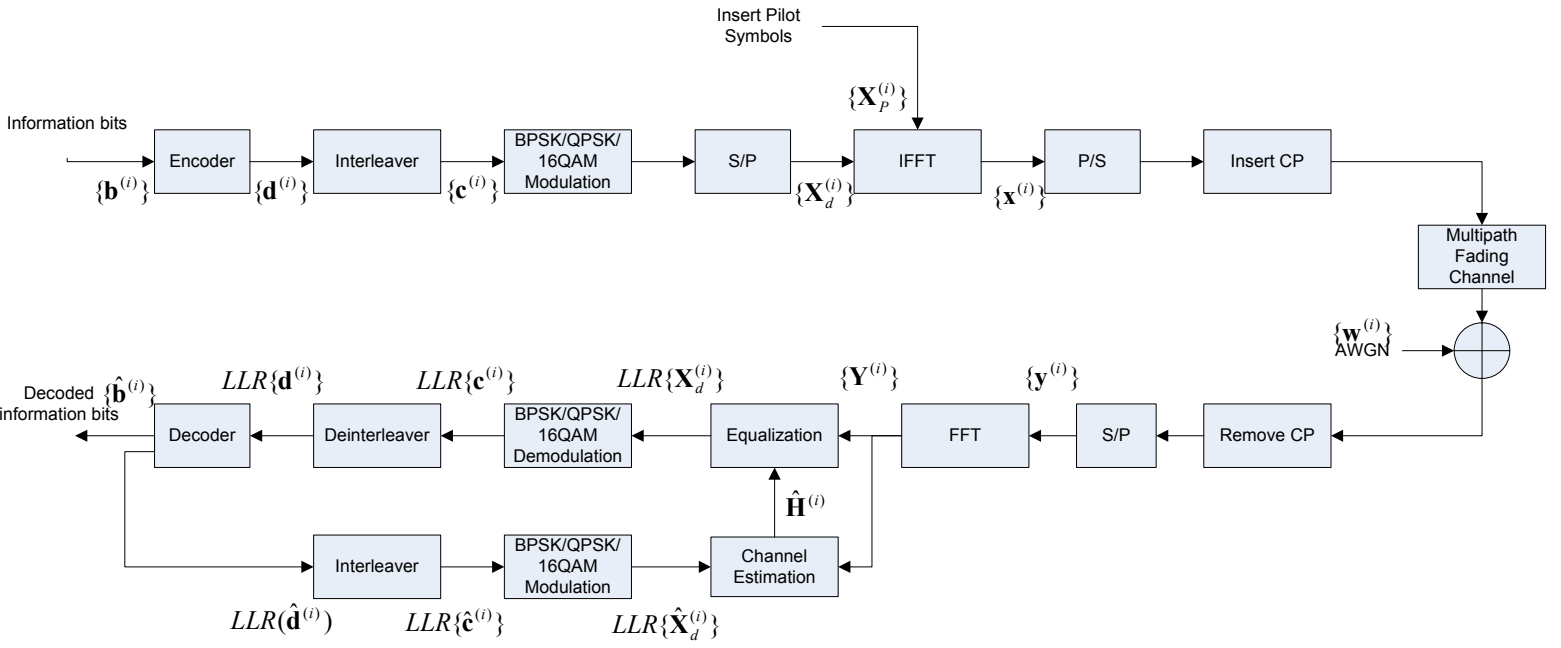

Fig. 2. OFDM system with iterative turbo channel estimation.

where the number of fading taps $L=\left\lceil\tau_{\max } / T_{s}\right\rceil$ is the maximum delay in terms of OFDM samples.

Assuming that the $\mathrm{CP}$ is longer or at least equal to the maximum channel delay spread $L$, i.e. $L \leq G$, after removing the $\mathrm{CP}$, the sampled received signal can be characterized in the following tapped-delay-line model [27]:

$$
y^{(i)}(n)=\sum_{l=0}^{L-1} h^{(i)}(n, l) x^{(i)}(n-l)+w^{(i)}(n),
$$

where $w^{(i)}(n)$ is the additive white Gaussian noise (AWGN) with zero mean and variance $\sigma_{w}^{2}$. In the range of $0 \leq n \leq$ $N-1$, the received signal $y^{(i)}(n)$ is immune to the interference by previous OFDM symbol due to the CP. The demodulated signal in the frequency domain is obtained by taking a $N$-point DFT of $y^{(i)}(n)$ as:

$$
\begin{aligned}
Y^{(i)}(m)= & \frac{1}{\sqrt{N}} \sum_{n=0}^{N-1} y^{(i)}(n) e^{-j 2 \pi m n / N}=H_{m, m}^{(i)} X^{(i)}(m) \\
& +\sum_{k \neq m}^{N-1} H_{m, k}^{(i)} X^{(i)}(k)+W^{(i)}(m),
\end{aligned}
$$

where

$$
\begin{gathered}
H_{m, m}^{(i)}=\frac{1}{N} \sum_{n=0}^{N-1} \sum_{l=0}^{L-1} h^{(i)}(n, l) e^{-j 2 \pi l m / N} \\
=\frac{1}{N} \sum_{n=0}^{N-1} \hbar_{m}^{(i)}(n) \\
H_{m, k}^{(i)}=\frac{1}{N} \sum_{n=0}^{N-1}\left\{\sum_{l=0}^{L-1} h^{(i)}(n, l) e^{-j 2 \pi l k / N}\right\} e^{-j 2 \pi(m-k) n / N} \\
=\frac{1}{N} \sum_{n=0}^{N-1} \hbar_{k}^{(i)}(n) e^{-j 2 \pi(m-k) n / N}
\end{gathered}
$$

and

$$
W^{(i)}(m)=\frac{1}{\sqrt{N}} \sum_{n=0}^{N-1} w^{(i)}(n) e^{-j 2 \pi m n / N},
$$

are the multiplicative distortion at the desired subcarrier $m$, the neighboring subcarrier $k$, and AWGN after DFT respectively. In (6), $\hbar_{m}^{(i)}(n)$ is the channel frequency response of subcarrier $m$ at time $n$ in $i^{\text {th }}$ OFDM symbol. If the channel is assumed to be time-invariant during one OFDM symbol period, $\hbar_{k}^{(i)}(n)$ is constant in (7), and $H_{m, k}^{(i)}$ vanishes. In this case, $Y^{(i)}(m)$ in (5) only contains the multiplicative distortion at the desired subcarrier, which can be easily compensated by a one-tap frequency domain equalizer.

Denote the received time-domain signal in (4) by a $N \times 1$ vector $\mathbf{y}^{(i)}=\left[y^{(i)}(0), y^{(i)}(1), \cdots, y^{(i)}(N-1)\right]^{T}$, the IDFT coefficients by a $N \times N$ matrix whose $(m, n)^{t h}$ element is $[\mathbf{F}]_{m, n}=e^{j 2 \pi m n / N} / \sqrt{N}$, AWGN as $N \times 1$ vector $\mathbf{w}^{(i)}=\left[w^{(i)}(0), w^{(i)}(1), \cdots, w^{(i)}(N-1)\right]^{T}$, and time-domain channel matrix by $N \times N$ matrix in (9), (4) can be expressed as:

$$
\mathbf{y}^{(i)}=\mathbf{h}^{(i)} \mathbf{F} \mathbf{X}^{(i)}+\mathbf{w}^{(i)}
$$

The received frequency-domain signal after DFT is given by:

$$
\begin{aligned}
\mathbf{Y}^{(i)} & =\mathbf{F}^{H} \mathbf{y}^{(i)}=\mathbf{F}^{H} \mathbf{h}^{(i)} \mathbf{F} \mathbf{X}^{(i)}+\mathbf{F}^{H} \mathbf{w}^{(i)} \\
& =\mathbf{H}^{(i)} \mathbf{X}^{(i)}+\mathbf{W}^{(i)},
\end{aligned}
$$

where $\mathbf{H}^{(i)}=\mathbf{F}^{H} \mathbf{h}^{(i)} \mathbf{F}$ and $\mathbf{W}^{(i)}=\mathbf{F}^{H} \mathbf{W}^{(i)}$. For a general time-varying channel, $\mathbf{H}^{(i)}$ has non-trivial off-diagonal elements $\left[\mathbf{H}^{(i)}\right]_{m, k}$ given by (7). Estimation of the entire $N \times N$ channel matrix incurs a prohibitive complexity. Therefore, only the diagonal coefficients of $\mathbf{H}^{(i)}$ are estimated in practice. The degradation caused by ignoring the off-diagonal intercarrier interference (ICI) terms in $\mathbf{H}^{(i)}$ can be evaluated by investigating the cross-correlation between elements in $\mathbf{H}^{(i)}$, which is given by:

$$
\begin{aligned}
E\left\{H_{r, s}^{(i)} \cdot\left(H_{p, q}^{(i)}\right)^{*}\right\}= & \frac{1}{N^{2}} \sum_{l=0}^{L-1} \alpha_{l} \cdot e^{-j 2 \pi(s-q) l / N} \\
& \cdot \sum_{n=0}^{N-1} \sum_{m=0}^{N-1} J_{0}\left[2 \pi f_{m}(n-m) T_{s}\right] \\
& \cdot e^{-j 2 \pi(r-s) n / N} e^{j 2 \pi(p-q) m / N}
\end{aligned}
$$




$$
\mathbf{h}^{(i)}=\left[\begin{array}{ccccccccc}
h_{0,0}^{(i)} & 0 & 0 & \ldots & 0 & h_{0, L-1}^{(i)} & h_{0, L-2}^{(i)} & \ldots & h_{0,1}^{(i)} \\
h_{1,1}^{(i)} & h_{1,0}^{(i)} & 0 & \ldots & 0 & 0 & h_{1, L-1}^{(i)} & \ldots & h_{1,2}^{(i)} \\
\vdots & \vdots & \vdots & \ddots & \ddots & \ddots & \ddots & \ddots & \vdots \\
0 & 0 & 0 & \ldots & h_{N-1, L-1}^{(i)} & h_{N-1, L-2}^{(i)} & \ldots & \ldots & h_{N-1,0}^{(i)}
\end{array}\right]
$$

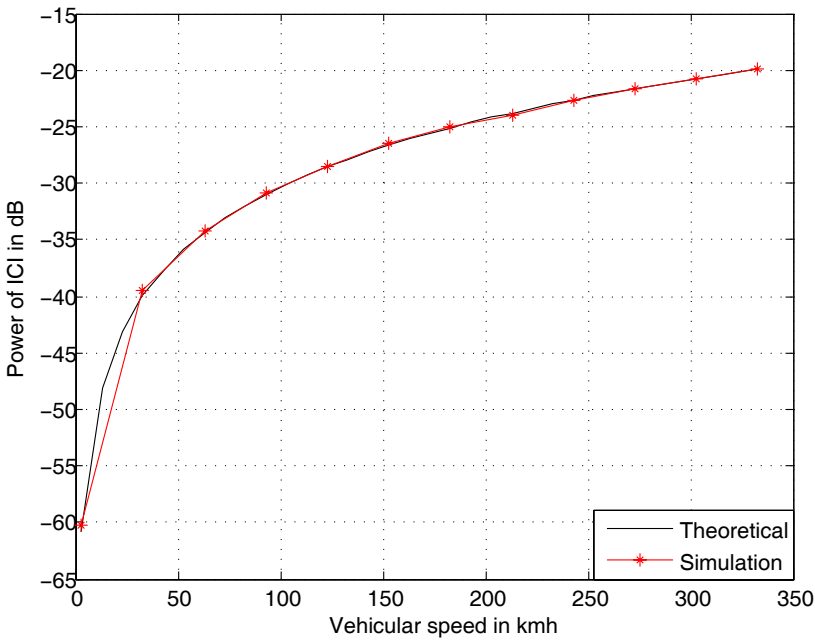

Fig. 3. ICI Power for IMT-2000 vehicular-A channel with central frequency of $5 \mathrm{GHz}$ and 256 subcarriers.

for $H_{r, s}^{(i)}$ and $H_{p, q}^{(i)}$. The power of ICI for a particular subcarrier $m$ is expressed as:

$$
\begin{aligned}
& P_{I C I}^{m}= E\left\{\left\|\sum_{k \neq m} H_{m, k}^{(i)} X^{(i)}(m)\right\|^{2}\right\}=\sum_{m \neq k}\left\|H_{m, k}^{(i)}\right\|^{2} \\
&= \frac{1}{N^{2}} \sum_{k \neq m} \sum_{l=0}^{L-1} \alpha_{l}\left\{N+2 \sum_{p=1}^{N-1}(N-p)\right. \\
&\left.\cdot J_{0}\left(2 \pi f_{m} p T_{s}\right) \cos \left[\frac{2 \pi(m-k) p}{N}\right]\right\}
\end{aligned}
$$

and the average power of ICI over all subcarriers is given by:

$$
\begin{aligned}
P_{I C I}= & \frac{1}{N} \sum_{m=0}^{N-1} P_{I C I}^{m} \\
= & \frac{N-1}{N}+\frac{4}{N^{3}} \sum_{l=0}^{L-1} \alpha_{l} \sum_{p=1}^{N-1}(N-p) J_{0}\left(2 \pi f_{m} p T_{s}\right) \\
& \cdot \sum_{q=1}^{N-1}(N-q) \cos \left(\frac{2 \pi p q}{N}\right),
\end{aligned}
$$

Fig. 3 shows average ICI Power for IMT-2000 vehicular-A channel [4] at various mobile speeds with central frequency of $5 \mathrm{GHz}$ and 256 subcarriers [2], [3]. It can be seen that ICI for the mobile channel in most practical Doppler spreads is below $-20 \mathrm{~dB}$. Therefore, we focus on the diagonal channel states in (6) and treat the ICI term as an additional embedded Gaussian noise, according to the central limit theorem.

\section{ITERATIVE RECEIVER OUTLINE}

The proposed receiver is shown in Fig. 2, where a channel estimator, a demapper module, and a maximum a posteriori
(MAP) decoder work in an iterative fashion. At each iteration, channel estimator estimates the channel frequency response based on the soft decoded data information from previous iteration, the demapper computes the a posteriori probability (APP's) $P\left(X_{d}^{(i)}(m) \mid Y^{(i)}(m), \tilde{H}_{m, m}^{(i)}\right) 0 \leq m \leq N-1$ given the channel estimates $\tilde{H}_{m, m}^{(i)}$ and received symbol $Y^{(i)}(m)$, and outputs extrinsic information for the coded bits in symbol $X_{d}^{(i)}(m)$. More specifically, the demapper outputs log likelihood ratio (LLR) $\lambda_{1}^{e}$ of the $k^{t h}$ coded bits $c_{k}$ in symbol $X_{d}^{(i)}(m)$ as in (15), where $U_{k}^{+}$is the constellation set that contains all the symbols whose $k^{t h}$ bit is 1 , and $U_{k}^{-}$is the constellation set that contains all the symbols whose $k^{\text {th }}$ bit is 0 . The conditional probability is computed as in (16).

The LLRs on the coded bits are de-interleaved and passed to the MAP decoder to be decoded. The MAP decoder feedbacks the extrinsic information $\lambda_{2}^{e}\left(c_{k}\left(X_{d}^{(i)}(m)\right)\right)$, which is used to compute the soft data symbol as follows:

$$
\begin{array}{r}
\hat{X}_{d}^{(i)}(m)=\sum_{s_{j} \in U(A)} s_{j} \cdot P\left(X_{d}^{(i)}(m)=s_{j}\right), \\
P\left(X_{d}^{(i)}(m)=s_{j}\right)=\prod_{l=1}^{\log _{2}|U(A)|} P\left(c_{l}\left(X_{d}^{(i)}(m)\right)\right),
\end{array}
$$

where $|U(A)|$ denotes the cardinality of the set $U(A)$. In the case of BPSK and Gray-coded QPSK, the soft data symbol can be obtained by:

$$
\begin{aligned}
\hat{X}_{d}^{(i)}(m)= & \tanh \left(\lambda_{2}^{e}\left(c\left(X_{d}^{(i)}(m)\right)\right) / 2\right) \\
\hat{X}_{d}^{(i)}(m)= & \frac{1}{\sqrt{2}}\left(\tanh \left(\lambda_{2}^{e}\left(c_{0}\left(X_{d}^{(i)}(m)\right)\right) / 2\right)\right. \\
& \left.+j \tanh \left(\lambda_{2}^{e}\left(c_{1}\left(X_{d}^{(i)}(m)\right)\right) / 2\right)\right) .
\end{aligned}
$$

The soft symbols will be used in channel estimation as detailed in the following section.

\section{Iterative Turbo ChANNEL Estimation}

In this section, we present a novel iterative channel estimation method with three distinctive operation stages, namely initial coarse estimation stage, iterative estimation stage, and a final estimation stage. Assuming that OFDM symbols are transmitted on a frame by frame basis. In the downlink transmission, each frame consists of one preamble followed by a number of data symbols. In each data symbol, pilots are evenly distributed across used subcarriers. In the uplink transmission, there is no preamble but only pilots. These configurations are specified in [2], [3].

\section{A. Initial Coarse Estimation Stage}

Initial coarse estimation stage is performed at the first iteration. The system model for pilot symbol transmission is 


$$
\begin{gathered}
\lambda_{1}^{e}\left(c_{k}\left(X_{d}^{(i)}(m)\right)\right)=\ln \frac{\sum_{s_{j} \in U_{k}^{+}(A)} P\left(X_{d}^{(i)}(m)=s_{j} \mid Y^{(i)}(m), \tilde{H}_{m, m}^{(i)}, \lambda_{2}^{e}\right)}{\sum_{s_{j} \in U_{k}^{-}(A)} P\left(X_{d}^{(i)}(m)=s_{j} \mid Y^{(i)}(m), \tilde{H}_{m, m}^{(i)}, \lambda_{2}^{e}\right)}, \\
P\left(X_{d}^{(i)}(m)=s_{j} \mid Y^{(i)}(m), \tilde{H}_{m, m}^{(i)}, \lambda_{2}^{e}\right)=\frac{1}{2 \pi \sigma_{w^{\prime}}^{2}} \exp \left(-\frac{\left\|Y^{(i)}(m)-\tilde{H}_{m, m}^{(i)} X_{d}^{(i)}(m)\right\|^{2}}{2 \sigma_{w^{\prime}}^{2}}\right) \prod_{l \neq k} P\left(c_{l}\left(X_{d}^{(i)}(m)\right)\right),
\end{gathered}
$$

given by:

$$
\begin{aligned}
Y^{(i)}(p)= & H_{p, p}^{(i)} \sqrt{E_{p}} X_{P}^{(i)}(p)+\sum_{q \neq p} H_{p, q}^{(i)} \sqrt{E_{p}} X_{P}^{(i)}(q) \\
& +\sum_{n \neq p, q} H_{p, n}^{(i)} \sqrt{E_{d}} X_{d}^{(i)}(n)+W^{(i)}(p),
\end{aligned}
$$

where $E_{p}$ and $E_{d}$ are the energy of pilot and data symbol, respectively. Channel frequency response at pilot subcarrier is obtained by the LS approach:

$$
\begin{aligned}
\tilde{H}_{p, p}^{(i)}= & Y^{(i)}(p) \frac{\left(X_{P}^{(i)}(p)\right)^{*}}{\sqrt{E_{p}}} \\
= & H_{p, p}^{(i)}+\sum_{q \neq p} H_{p, q}^{(i)} X_{P}^{(i)}(q)\left(X_{P}^{(i)}(p)\right)^{*} \\
& +\sum_{n \neq p, q} H_{p, n}^{(i)} \sqrt{\frac{E_{d}}{E_{p}}} X_{d}^{(i)}(n)\left(X_{P}^{(i)}(p)\right)^{*} \\
& +\frac{W^{(i)}(p)\left(X_{P}^{(i)}(p)\right)^{*}}{\sqrt{E_{p}}} \\
= & H_{p, p}^{(i)}+W_{P}^{(i)}(p),
\end{aligned}
$$

Assuming pilots and data symbols are independent, it can be shown that $W_{P}^{\prime(i)}(p)$ is $\mathcal{N}\left(0, \frac{\sigma_{w}^{2}+\sigma_{I C I}^{2}}{E_{p}}=\frac{\sigma_{w^{\prime}}}{E_{p}}\right)$.

For OFDM data symbols, channel tracking is applied to obtain initial coarse channel estimates. In the downlink transmission, channel estimates for the $i^{t h}$ symbol is given by:

$$
\tilde{\mathbf{H}}^{(i)}=\tilde{\mathbf{H}}^{(i-1)}+\boldsymbol{f i l t}\left(\tilde{\mathbf{H}}_{\mathbf{p}}^{(i)}-\tilde{\mathbf{H}}_{\mathbf{p}}^{(i-1)}\right),
$$

where $\tilde{\mathbf{H}}_{\mathbf{p}}$ is the channel estimates at pilot subcarriers. filt $(\cdot)$ denotes the interpolation filter, which can be FFT based, MMSE based, or linear interpolation based. In this paper, linear interpolation is employed due to its low complexity. More specifically, assuming the pilot spacing is $\delta, \tilde{H}_{p, p}^{(i)}$ and $\tilde{H}_{p+\delta, p+\delta}^{(i)}$ are the channel estimates from two adjacent pilots. The channel estimate $\tilde{H}_{m, m}^{(i)}$ at subcarrier $m$, which is between pilot subcarrier $p$ and $p+\delta$ is given by:

$$
\tilde{H}_{m, m}^{(i)}=\left[\begin{array}{ll}
1-\frac{m-p}{\delta} & \frac{m-p}{\delta}
\end{array}\right] \cdot\left[\begin{array}{c}
\tilde{H}_{p, p}^{(i)} \\
\tilde{H}_{p+\delta, p+\delta}^{(i)}
\end{array}\right],
$$

In contrast, in the uplink transmission, the initial channel estimates for the $i^{t h}$ symbol is given by:

$$
\tilde{\mathbf{H}}^{(i)}=\operatorname{filt}\left(\tilde{\mathbf{H}}_{\mathbf{p}}^{(i)}\right),
$$

where the design of the interpolation filter is based on the pilots allocation in practical OFDM systems. After initial coarse channel estimates, data detection is performed in the demapper module.

\section{B. Iterative Estimation Stage}

In the iterative estimation stage, LS estimation is first performed for both pilot and data subcarriers, followed by frequency-domain combining and time-domain combining. Similar to the pilot tones, the system model for data symbol transmission is given by:

$$
\begin{aligned}
Y^{(i)}(m)= & H_{m, m}^{(i)} \sqrt{E_{d}} X_{d}^{(i)}(m)+\sum_{n \neq m} H_{m, n}^{(i)} \sqrt{E_{d}} X_{d}^{(i)}(n) \\
& +\sum_{p \neq m} H_{m, p}^{(i)} \sqrt{E_{p}} X_{P}^{(i)}(p)+W^{(i)}(m),
\end{aligned}
$$

Because the data detection in previous iterations may not be reliable, the energy of soft decoded data symbol in (19) and (20) may be less than 1 . If the soft decoded data symbol is directly applied to LS estimation, the channel estimates are subject to a bias due to the imperfect decoding information. To overcome this problem, channel estimate at the $m^{\text {th }}$ subcarrier is normalized by the average energy of the soft decoded data symbols within a moving average window (MAW) as:

$$
\begin{aligned}
\tilde{H}_{m, m}^{(i)}= & Y^{(i)}(m) \frac{\left(\hat{X}_{d}^{(i)}(m)\right)^{*}}{\sqrt{E_{d}\left\|\hat{X}_{d \in M A W}^{(i)}\right\|^{2}}} \\
= & H_{m, m}^{(i)} \frac{X_{d}^{(i)}(m)}{\sqrt{\left\|\hat{X}_{d \in M A W}^{(i)}\right\|^{2}}}\left(\hat{X}_{d}^{(i)}(m)\right)^{*} \\
& +\sum_{n \neq m} H_{m, n}^{(i)} \frac{X_{d}^{(i)}(n)}{\sqrt{\left\|\hat{X}_{d \in M A W}^{(i)}\right\|^{2}}}\left(\hat{X}_{d}^{(i)}(m)\right)^{*} \\
& +\sum_{p \neq m} H_{m, p}^{(i)} \frac{\sqrt{E_{p}} X_{P}^{(i)}(p)}{\sqrt{E_{d}\left\|\hat{X}_{d \in M A W}^{(i)}\right\|^{2}}}\left(\hat{X}_{d}^{(i)}(m)\right)^{*} \\
& +\frac{1}{\sqrt{E_{d}\left\|\hat{X}_{d \in M A W}^{(i)}\right\|^{2}}} W^{(i)}(m)\left(\hat{X}_{d}^{(i)}(m)\right)^{*} \\
\approx & H_{m, m}^{(i)} \sqrt{\left\|\hat{X}_{d \in M A W}^{(i)}\right\|^{2}}+W_{d}^{(i)}(m),
\end{aligned}
$$

where

$$
\left\|\hat{X}_{d \in M A W}^{(i)}\right\|^{2}=E\left\{\hat{X}_{d \in M A W}^{(i)}(m)\left(\hat{X}_{d \in M A W}^{(i)}(m)\right)^{*}\right\},
$$

is the average energy of soft coded data information in the MAW. With the similar assumptions in the pilot estimation, it can be shown that $W_{d}^{\prime(i)}(m)$ is $\mathcal{N}\left(0, \frac{\sigma_{w}^{2}+\sigma_{I C I}^{2}}{E_{d}}=\frac{\sigma_{w^{\prime}}^{2}}{E_{d}}\right)$.

Due to the correlation in the frequency domain, low pass filtering can be performed by combining channel estimates from both pilot tones and soft coded data information within the MAW to generate improved channel estimates. The size of the MAW is determined by the system coherent bandwidth 
and subcarrier spacing ${ }^{1}$. Assuming within the MAW, the channel frequency response is highly correlated, i.e. $H_{p, p}^{(i)} \approx$ $H_{d, d}^{(i)} \approx H_{m, m}^{(i)}$, the weighted average for the channel frequency response at subcarrier $m$ is given by:

$$
\begin{aligned}
\hat{H}_{m, m}^{(i)}= & \omega_{p} \sum_{p \in M A W} \tilde{H}_{p, p}^{(i)}+\omega_{d} \sum_{d \in M A W} \tilde{H}_{d, d}^{(i)} \\
= & \omega_{p} \sum_{p \in M A W}\left(H_{m, m}^{(i)}+W_{P}^{(i)}\right) \\
& +\omega_{d} \sum_{d \in M A W}\left(H_{m, m}^{(i)} \sqrt{\left\|\hat{X}_{d \in M A W}^{(i)}\right\|^{2}}+W_{d}^{(i)}\right) \\
= & \left(N_{p} \omega_{p}+N_{d} \omega_{d} \sqrt{\left\|\hat{X}_{d \in M A W}^{(i)}\right\|^{2}}\right) H_{m, m}^{(i)}{ }^{\prime} \\
& +\underbrace{\left(\omega_{p} W_{P}^{(i)}+\omega_{d} \sum_{d \in M A W} W_{d}^{(i)}\right)}_{\mathcal{N}\left(0, N_{p} \omega_{p}^{2} \frac{\sigma_{w}^{2}}{E_{p}}+N_{d} \omega_{d}^{2} \frac{\sigma_{w}^{2}}{E_{d}}\right)},(29)
\end{aligned}
$$

where $N_{p}$ and $N_{d}$ are the number of pilot and data symbols within the MAW. The optimal weight values $\left\{\omega_{p}, \omega_{d}\right\}$, can be determined using the Maximum Ratio Combining (MRC) principle, which is mathematically formulated into the following Lagrange multiplier problem:

$$
\begin{aligned}
\left\{\omega_{p}, \omega_{d}\right\}= & \arg \min _{\omega_{p}, \omega_{d}}\left\{\left(N_{p} \omega_{p}^{2} \frac{\sigma_{w^{\prime}}^{2}}{E_{p}}+N_{d} \omega_{d}^{2} \frac{\sigma_{w^{\prime}}^{2}}{E_{d}}\right)+\lambda\left(N_{p} \omega_{p}\right.\right. \\
& \left.\left.+N_{d} \omega_{d} \sqrt{\left\|\hat{X}_{d \in M A W}^{(i)}\right\|^{2}}-1\right)\right\}
\end{aligned}
$$

where $\lambda$ is the Lagrange multiplier. Hence, the optimal weights $\left\{\omega_{p}, \omega_{d}\right\}$ can be derived as:

$$
\begin{aligned}
\omega_{p} & =\frac{1}{N_{p}+N_{d} \frac{E_{d}}{E_{p}}\left\|\hat{X}_{d \in M A W}^{(i)}\right\|^{2}}, \\
\omega_{d} & =\frac{\sqrt{\left\|\hat{X}_{d \in M A W}^{(i)}\right\|^{2}}}{N_{p} \frac{E_{p}}{E_{d}}+N_{d}\left\|\hat{X}_{d \in M A W}^{(i)}\right\|^{2}} .
\end{aligned}
$$

To further improve channel estimates, time domain MAW combining is applied to the channel frequency response. Based on the coherence time of the channel for practical OFDM system, it is observed that OFDM channel frequency responses are highly correlated in the time domain [28] for consecutive OFDM symbols, i.e. $H_{m, m}^{(i-1)} \approx H_{m, m}^{(i)}$. Therefore, another $\mathrm{MRC}$ can be performed, which is given by:

$$
\begin{aligned}
& \hat{\hat{H}}_{m, m}^{(i)}=\alpha \hat{H}_{m, m}^{(i-1)}+\beta \hat{H}_{m, m}^{(i)}=(\alpha+\beta) H_{m, m}^{(i)} \\
& +\underbrace{\left(\alpha W^{\prime \prime}(i-1)(m)+\beta W^{\prime \prime}(i)(m)\right)}_{\mathcal{N}\left(0, \alpha^{2} \sigma_{w^{\prime \prime}(i-1)}^{2}+\beta^{2} \sigma_{w^{\prime \prime}(i)}^{2}\right)} .
\end{aligned}
$$

In (33), $\alpha$ and $\beta$ are weighting parameters obtained by minimizing the estimation error, that is:

$$
\{\alpha, \beta\}=\underset{\alpha, \beta}{\operatorname{argmin}}\left\{\left(\alpha^{2} \sigma_{w^{\prime \prime}(i-1)}^{2}+\beta^{2} \sigma_{w^{\prime \prime}(i)}^{2}\right)+\lambda(\alpha+\beta-1)\right\},
$$

\footnotetext{
${ }^{1}$ In the system configuration as mentioned in Section VII, the subcarrier spacing is $5.12 \mathrm{kHz}$, while the coherent bandwidth is $54 \mathrm{kHz}$ for the channel considered in this paper. Therefore, it is reasonable to define a MAW of size 9.
}

which are given by:

$$
\begin{aligned}
& \alpha=\frac{\sigma_{w^{\prime \prime}(i)}^{2}}{\sigma_{w^{\prime \prime}(i-1)}^{2}+\sigma_{w^{\prime \prime}(i)}^{2}}, \\
& \beta=\frac{\sigma_{w^{\prime \prime}(i-1)}^{2}}{\sigma_{w^{\prime \prime}(i-1)}^{2}+\sigma_{w^{\prime \prime}(i)}^{2}} .
\end{aligned}
$$

The advantage of the proposed method is that the weights in (31), (32), (35) and (36) are adaptive to the number and power of pilots and data symbols in the MAW, and most importantly, the reliability of decoding data information in the specific OFDM symbols. Therefore, the combining is performed in perfect proportion to the available information. As the iterations proceed, the available a priori information on data signals improves, the weights associated with the data-aided channel estimates increase accordingly, where the decoding data serve as virtual reference signals.

\section{Final Estimation Stage}

The final estimation stage is performed on the final iteration, where the decoding information from MAP decoder becomes very reliable, and can serve almost as good as reference signals. For an OFDM symbol full of virtual reference signals, maximum likelihood estimator (MLE) or MMSE estimator is able to provide a further improvement over the LS based MRC channel estimator in previous section. To see this clearly, we reformulate the OFDM system channel estimation model in (11) as:

$$
\mathbf{Y}^{(i)}=\mathbf{X}^{\prime(i)} \mathbf{H}^{\prime(i)}+\mathbf{W}^{\prime(i)}
$$

where $\mathbf{X}^{\prime(i)}=\operatorname{diag}\left(X_{0}^{(i)}, X_{1}^{(i)}, \ldots, X_{N-1}^{(i)}\right)$ is the $N \times N$ diagonal matrix with assumption that pilot and data symbols are taken from a PSK constellation with unit energy, i.e., $\left\|X_{m}^{(i)}\right\|=1$. $\mathbf{H}^{\prime(i)}$ is the $N \times 1$ channel frequency response vector under investigation, and $\mathbf{W}^{\prime}(i)$ is the equivalent $N \times 1$ noise vector with $\sigma_{w^{\prime}}^{2}=\sigma_{w}^{2}+\sigma_{I C I}^{2}$. If $\mathbf{X}^{\prime(i)}$ is perfectly known, the LS estimation is given by:

$$
\begin{aligned}
\tilde{\mathbf{H}}_{L S}^{\prime(i)} & =\left[\left(\mathbf{X}^{\prime}(i)\right)^{H} \mathbf{X}^{\prime}(i)\right]^{-1}\left(\mathbf{X}^{\prime}(i)\right)^{H} \mathbf{Y}^{(i)} \\
& =\mathbf{H}^{\prime(i)}+\left[\left(\mathbf{X}^{\prime}(i)\right)^{H} \mathbf{X}^{\prime(i)}\right]^{-1}\left(\mathbf{X}^{\prime(i)}\right)^{H} \mathbf{W}^{\prime}(i) \\
& =\mathbf{H}^{\prime(i)}+\left(\mathbf{X}^{\prime(i)}\right)^{-1} \mathbf{W}^{\prime(i)},
\end{aligned}
$$

where $\left(\mathbf{X}^{\prime(i)}\right)^{-1} \mathbf{W}^{\prime}(i)$ is statistically equivalent to $\mathbf{W}^{\prime}(i)$ for a PSK constellation. Hence, a new signal model [29] based on (38) is used for estimating $\mathbf{H}^{\prime}(i)$, given by:

$$
\begin{aligned}
& \tilde{\mathbf{H}}_{L S}^{\prime(i)}=\mathbf{H}^{\prime}(i)+\left[\left(\mathbf{X}^{\prime}(i)\right)^{H} \mathbf{X}^{\prime}(i)\right]^{-1}\left(\mathbf{X}^{\prime}(i)\right)^{H} \mathbf{W}^{\prime}(i) \\
& =\mathbf{G h}^{\prime}{ }^{(i)}+\left[\left(\mathbf{X}^{\prime}(i)\right)^{H} \mathbf{X}^{\prime(i)}\right]^{-1}\left(\mathbf{X}^{\prime}(i)\right)^{H} \mathbf{W}^{\prime(i)} \\
& =\mathbf{G} \mathbf{h}^{\prime(i)}+\left(\mathbf{X}^{\prime(i)}\right)^{-1} \mathbf{W}^{\prime}(i) \text {, }
\end{aligned}
$$

where $\mathbf{G}$ is the $N \times L$ matrix with element $[\mathbf{G}]_{n, l}=$ $e^{-j 2 \pi n l / N}, 0 \leq n \leq N-1$ and $0 \leq l \leq L-1$. $\mathbf{h}^{\prime}(i)=\left[h_{0}{ }^{(i)}, h_{1}^{\top}(i), \cdots, h_{L-1}^{\prime(i)}\right]^{T}$ is a $L \times 1$ channel impulse response vector, where $h_{n}^{\prime(i)}$ is given by:

$$
h_{l}^{\prime}{ }^{(i)}=\frac{1}{N} \sum_{n=0}^{N-1} h^{(i)}(n, l),
$$


If $\mathbf{H}^{\prime}(i)$ is assumed to be a deterministic and unknown vector, the MLE can be derived following the invariance property [30], given by:

$$
\begin{aligned}
\hat{\mathbf{H}}_{\mathbf{M L E}}^{\prime(i)} & =\mathbf{G}\left(\mathbf{G}^{H} \mathbf{G}\right)^{-1} \mathbf{G}^{H} \tilde{\mathbf{H}}_{L S}^{(i)} \\
& =\mathbf{G}\left(\mathbf{G}^{H} \mathbf{G}\right)^{-1} \mathbf{G}^{H}\left[\left(\mathbf{X}^{\prime(i)}\right)^{H} \mathbf{X}^{\prime(i)}\right]^{-1}\left(\hat{\mathbf{X}}^{\prime(i)}\right)^{H} \mathbf{Y}^{(i)} \\
& =\frac{1}{N} \mathbf{G} \mathbf{G}^{H}\left(\hat{\mathbf{X}}^{\prime(i)}\right)^{-1} \mathbf{Y}^{(i)}
\end{aligned}
$$

where $\hat{\mathbf{X}}^{\prime(i)}$ is soft coded OFDM symbol from the last second iteration with pilot tones.

As $\mathbf{H}^{\prime}(i)$ is random in nature, Bayesian estimators are able to improve the performance by exploiting the priori knowledge on channel statistics. In this paper, we consider the MMSE estimator [29], given by:

$$
\begin{aligned}
& \hat{\mathbf{H}}_{\text {MMSE }}^{\prime(i)}=\mathbf{G R}_{\mathbf{h}^{\prime} \mathbf{h}^{\prime}}\left(\mathbf{G}^{H} \mathbf{G R}_{\mathbf{h}^{\prime} \mathbf{h}^{\prime}}+\sigma_{w^{\prime}}^{2} \mathbf{I}_{\mathbf{L}}\right)^{-1} \mathbf{G}^{H} \tilde{\mathbf{H}}_{L S}^{\prime(i)} \\
& =\mathbf{G R}_{\mathbf{h}^{\prime} \mathbf{h}^{\prime}}\left(N \mathbf{R}_{\mathbf{h}^{\prime} \mathbf{h}^{\prime}}+\sigma_{w^{\prime}}^{2} \mathbf{I}_{\mathbf{L}}\right)^{-1} \mathbf{G}^{H} \tilde{\mathbf{H}}^{\prime(i)} \\
& =\mathbf{G R}_{\mathbf{h}^{\prime} \mathbf{h}^{\prime}}\left(N \mathbf{R}_{\mathbf{h}^{\prime} \mathbf{h}^{\prime}}+\sigma_{w^{\prime}}^{2} \mathbf{I}_{\mathbf{L}}\right)^{-1} \\
& \cdot \mathbf{G}^{H}\left(\hat{\mathbf{X}}^{\prime(i)}\right)^{-1} \mathbf{Y}^{(i)},
\end{aligned}
$$

where $\mathbf{R}_{\mathbf{h}^{\prime} \mathbf{h}^{\prime}}=E\left\{\mathbf{h}^{\prime} \mathbf{h}^{\prime H}\right\}=\operatorname{diag}\left(\alpha_{l}\right)$ is the $L \times L$ covariance matrix of $\mathbf{h}^{\prime}$ based on the WSSUS assumption. $\mathbf{I}_{\mathbf{L}}$ is the $L \times L$ identity matrix, and $\mathbf{G}^{H} \mathbf{G}=N \mathbf{I}_{\mathbf{L}}$.

\section{Mean Square Error Analysis of Iterative TURBO CHANNEL ESTIMATION}

In this section, we derive the lower bound of MSE for MLE and MMSE methods. In both methods, the MSE lower bounds are calculated for MLE and MMSE estimators in section IV-C. Extended from (41), the MLE can be expressed as:

$$
\hat{\mathbf{H}}_{\mathbf{M L E}}^{\prime(i)}=\mathbf{H}^{\prime(i)}+\mathbf{G}\left(\mathbf{G}^{H} \mathbf{G}\right)^{-1} \mathbf{G}^{H}\left(\mathbf{X}^{\prime(i)}\right)^{-1} \mathbf{W}^{\prime(i)},
$$

whose mean can be obtained as $E\left\{\left(\hat{\mathbf{H}}_{\mathbf{M L E}}^{\prime(i)}\right)\right\}=\mathbf{H}^{\prime(i)}$, and the covariance matrix can be obtained as:

$$
\begin{aligned}
C_{\hat{\mathbf{H}}_{\mathrm{MLE}}^{\prime(i)}} & =E\left\{\left(\hat{\mathbf{H}}_{\mathrm{MLE}}^{\prime(i)}-\mathbf{H}^{\prime(i)}\right)\left(\hat{\mathbf{H}}_{\mathrm{MLE}}^{\prime(i)}-\mathbf{H}^{\prime(i)}\right)^{H}\right\} \\
& =\sigma_{w^{\prime}}^{2} \mathbf{G}\left(\left(\mathbf{G}^{H} \mathbf{G}\right)^{-1}\right)^{H} \mathbf{G}^{H} \\
& =\frac{\sigma_{w^{\prime}}^{2}}{N} \mathbf{G G}^{H},
\end{aligned}
$$

where $\left(\mathbf{X}^{\prime}(i)\right)^{-1}\left(\left(\mathbf{X}^{\prime}(i)\right)^{-1}\right)^{H}=\mathbf{I}_{N}$ for PSK constellation considered in this paper. Hence, the corresponding MSE for MLE is given by:

$M S E_{M L E}=\frac{1}{N} \operatorname{Tr}\left(C_{\hat{\mathbf{H}}_{\mathrm{MLE}}^{\prime(i)}}\right)=\frac{1}{N} \operatorname{Tr}\left(\frac{\sigma_{w^{\prime}}^{2}}{N} \mathbf{G G}^{H}\right)=\frac{\sigma_{w^{\prime}}^{2} L}{N_{(45)}}$,

where $\operatorname{Tr}(\cdot)$ is the trace operation.

Similarly, for the MMSE estimator, the covariance matrix of $\hat{\mathbf{H}}_{\text {MMSE }}^{(i)}$ is given by:

$$
\begin{aligned}
C_{\hat{\mathbf{H}}_{\text {MMSE }}^{\prime(i)}} & =E\left\{\hat{\mathbf{H}}_{\mathrm{MMSE}}^{\prime(i)}\left(\hat{\mathbf{H}}_{\mathrm{MMSE}}^{\prime(i)}\right)^{H}\right\} \\
& =\sigma_{w^{\prime}}^{2} \mathbf{G R}_{\mathbf{h}^{\prime} \mathbf{h}^{\prime}}\left(N \mathbf{R}_{\mathbf{h}^{\prime} \mathbf{h}^{\prime}}+\sigma_{w^{\prime}}^{2} \mathbf{I}_{\mathbf{L}}\right)^{-1} \mathbf{G}^{H}(46)
\end{aligned}
$$

and the Bayesian MSE is given by:

$$
\begin{aligned}
M S E_{M M S E} & =\frac{1}{N} \operatorname{Tr}\left(C_{\hat{\mathbf{H}}_{\mathbf{M M S E}}^{\prime(i)}}\right) \\
& =\sigma_{w^{\prime}}^{2} \operatorname{Tr}\left\{\mathbf{R}_{\mathbf{h}^{\prime} \mathbf{h}^{\prime}}\left(N \mathbf{R}_{\mathbf{h}^{\prime} \mathbf{h}^{\prime}}+\sigma_{w^{\prime}}^{2} \mathbf{I}_{\mathbf{L}}\right)^{-1}\right\} \\
& =\frac{\sigma_{w^{\prime}}^{2}}{N} \operatorname{Tr}\left\{\operatorname{diag}\left(\frac{\alpha_{l}}{\alpha_{l}+\sigma_{w^{\prime}}^{2} / N}\right)\right\} \\
& =\frac{\sigma_{w^{\prime}}^{2}}{N} \sum_{l=0}^{L-1} \frac{1}{1+\sigma_{w^{\prime}}^{2} /\left(N \alpha_{l}\right)}
\end{aligned}
$$

MSEs in (45) and (47) will be used as benchmarks to evaluate the performance for proposed channel estimator in the following section. It can be shown that $M S E_{M M S E} \leq$ $M S E_{M L E}$ as the MMSE estimator utilizes channel statistical information in the estimation to enhance the performance.

\section{COMPlexity ANALYSis}

In this paper, the computational complexity of the proposed iterative turbo channel estimation is evaluated by the number of complex multiplications. Although the MAP decoder has significant computational complexity, this complexity exists regardless of the channel estimation scheme chosen. We therefore analyze only the complexity of the channel estimation techniques.

Assuming there are altogether $M$ iterations, and $N$ subcarriers. In the initial estimation stage, pilot estimation requires $N_{p}$ complex multiplications. To obtain the coarse channel frequency response at data tones, the linear interpolation between pilot tones requires $\left(N-N_{p}\right)$ complex multiplications.

In the iterative estimation stage, every iteration requires the same computational complexity. More specifically, in each iteration, the soft data channel estimation requires $\left(N-N_{p}\right)$ complex multiplications. The calculation of $\omega_{p}, \omega_{d}$ coefficients requires $N \times N_{M A W}^{F D}$ multiplications, frequency-domain filtering requires $N \times N_{M A W}^{F D}$ complex multiplications, where $N_{M A W}^{F D}$ is the frequency-domain MAW size. The calculation of $\alpha, \beta$ coefficients is real multiplication, hence it is ignored because the complexity to do real multiplication is considerably lower. The time-domain filtering requires $2 N$ complex multiplications. Therefore, there are totally $(M-2) \times(3 N-$ $\left.N_{p}+2 N \times N_{M A W}^{F D}\right)$ complex multiplications.

In the final estimation stage, only soft data channel estimation and MLE or MMSE operation are performed. Similar to iterative estimation stage, soft data channel estimation requires $\left(N-N_{p}\right)$ complex multiplications. MLE operation requires $O\left(N^{2}\right)$ complex multiplications and MMSE operation requires $O\left(N^{3}\right)$. Therefore, the total complexity is $N+O\left(N^{2}\right)$ for MLE and $N+O\left(N^{3}\right)$ for MMSE estimator. Table I summarizes the number of complex multiplications involved in each stage. It can be seen that if the final estimation stage is excluded, the complexity of initial coarse estimation stage and iterative estimation stage is $N+(M-2) \times\left(3 N-N_{p}+\right.$ $2 N \times N_{M A W}^{F D}$, which is in the order of $O(N)$. Compared to conventional MLE or MMSE estimation, the additional complexity from iterative channel estimation remains low. 
TABLE I

NUMBER OF COMPLEX MULTIPLICATIONS

\begin{tabular}{|c|c|c|c|}
\hline Operations & First Stage & Second Stage (per iteration) & Final Stage \\
\hline Pilot Estimation & $N_{p}$ & 0 & 0 \\
\hline Soft Data Estimation & 0 & $N-N_{p}$ & $N-N_{p}$ \\
\hline Linear Interpolation & $\left(N-N_{p}\right)$ & 0 & 0 \\
\hline$\omega_{p}, \omega_{d}$ Calculation & 0 & $N \times N_{M A W}^{F D}$ & 0 \\
\hline Frequency-domain Filtering & 0 & $N \times N_{M A W}^{F D}$ & 0 \\
\hline Time-domain Filtering & 0 & $2 * N$ & 0 \\
\hline ML Estimation & 0 & 0 & $O\left(N^{2}\right)$ \\
\hline MMSE Estimation & 0 & 0 & $O\left(N^{3}\right)$ \\
\hline Total for each stage & $O(N)$ & $O(N)$ & $O\left(N^{2}\right) o r O\left(N^{3}\right)$ \\
\hline
\end{tabular}

\section{Simulation Results}

\section{A. Simulation Setup}

In this section, an OFDM system with $N=256$ subcarriers, and 8 pilot tones as in [3] is considered. The carrier frequency is $5 \mathrm{GHz}$, and the bandwidth is $5 \mathrm{MHz}$. The subcarrier spacing is approximately $5.12 \mathrm{kHz}$. The IMT-2000 vehicular-A channel [4] is generated by Jakes model [26], with exponential decayed power profile $\{0,-1,-9,-10,-15,-20\}$ in $\mathrm{dB}$ and relative path delay $\{0,310,710,1090,1730,2510\}$ in ns. The coherent bandwidth is approximately $54 \mathrm{kHz}$. The frequency-domain MAW size is set to 9 to ensure that the correlation of channel frequency response within the window is sufficiently high. Unless stated otherwise, the vehicular speed is $333 \mathrm{kmh}$, which is translated to a Doppler frequency of $f_{m}=1540.125 \mathrm{~Hz}$. The $\mathrm{CP}$ duration is 16 samples. A rate- $1 / 2(5,7)_{8}$ convolutional code is used for channel coding. Random interleaving is adopted in the simulation and the modulation scheme is QPSK. The maximum number of iterations is set to 6. There are ten OFDM symbols per frame transmission. The energy of the pilot symbols is same as data symbols. Pilot tones are evenly distributed across subcarriers.

For the downlink transmission, the OFDM receiver with proposed iterative turbo channel estimation technique is compared to the OFDM receiver with preamble-based channel estimation [31] and iterative data derived channel estimation [5]. For the uplink transmission, the proposed receiver is compared to the conventional OFDM receiver with pilot-aided channel estimation [30], [31] using $P=64$ pilot tones. Hereafter, the iterative turbo MLE channel estimation and MMSE channel estimation refer to the iterative methods with ML estimation and MMSE estimation performed in the last iteration respectively. Performance comparisons are made in terms of frame error rate (FER) and channel estimation MSE. Performance of MSE will be compared to lower bounds for MLE and MMSE estimators respectively, which are derived in Section V. In addition to the $333 \mathrm{kmh}$ vehicular speed case, the performances of the proposed receiver at $120 \mathrm{kmh}$ and $60 \mathrm{kmh}$ are presented. Furthermore, the effect of carrier frequency offset (CFO) is considered as in the realistic OFDM systems [2], [3] due to channel delays and the difference between transmitter and receiver oscillators. Residual CFO of up to
$4 \%$ of the subcarrier spacing is present after synchronization and acquisition, which causes ICI and degrades the system performance.

\section{B. Numerical Results}

Fig. 4 shows the downlink performance of the proposed receiver over a number of iterations altogether with that using conventional preamble channel estimation and data derived channel estimation. The conventional receiver with just preamble estimation fails at such high mobility. The OFDM receiver with data derived channel estimation performs much better than the conventional preamble estimation, while the OFDM receiver with the proposed iterative channel estimation achieves the best performance among the three, and approaches that with perfect channel state information (CSI). As shown in Fig. 4(b), in the last iteration, the MSE of proposed iterative turbo channel estimation approaches MLE lower bound. This verifies the observations shown in Fig. 4(a).

Fig. 5 shows the uplink performance of the proposed receiver together with that using conventional pilot-aided channel estimation. Eight pilots are embedded in each OFDM symbol for the proposed system while 64 subcarriers are used for pilots in the other. In the first iteration, the proposed channel estimator has poor performance due to fewer pilots available for initial coarse estimation stage. However, as decoded soft data symbols are available for channel estimation in the later iterations, the iterative channel estimator outperforms the pilot-aided estimation with 64 inserted pilots. This demonstrates the advantage of proposed channel estimator in both SNR and throughput. The mean square error for both methods shown in Fig. 5(b) confirms the channel estimator performance. Fig. 6 shows the uplink performance for the proposed receiver and that with pilot-aided MLE/MMSE channel estimation. It can be seen that the proposed iterative turbo channel estimation performs $1 \mathrm{~dB}$ better with much fewer pilots. This observation shows that in high Doppler environment, the proposed OFDM receiver maintains the system throughput and has a SNR gain over advanced channel estimation filters.

Fig. 7 shows the FER performance comparison for various vehicular speeds. The performance improves for receivers with preamble based and data derived channel estimation as 


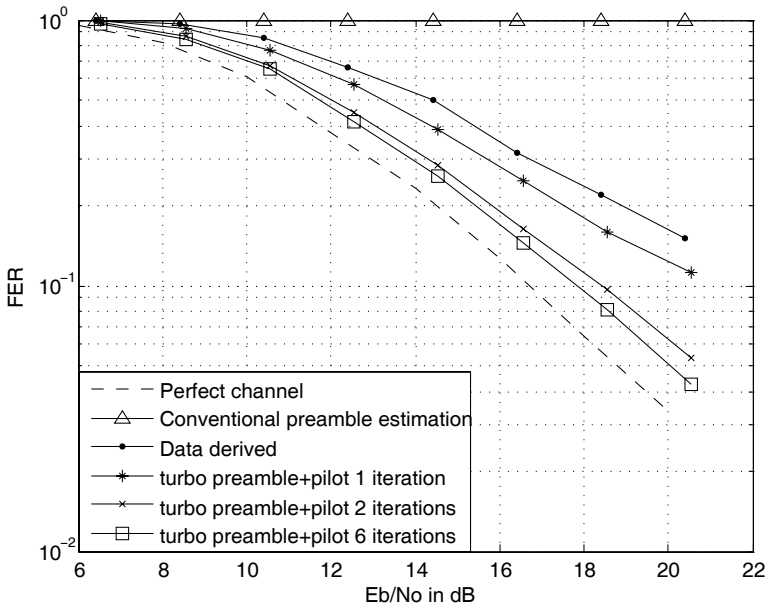

(a)

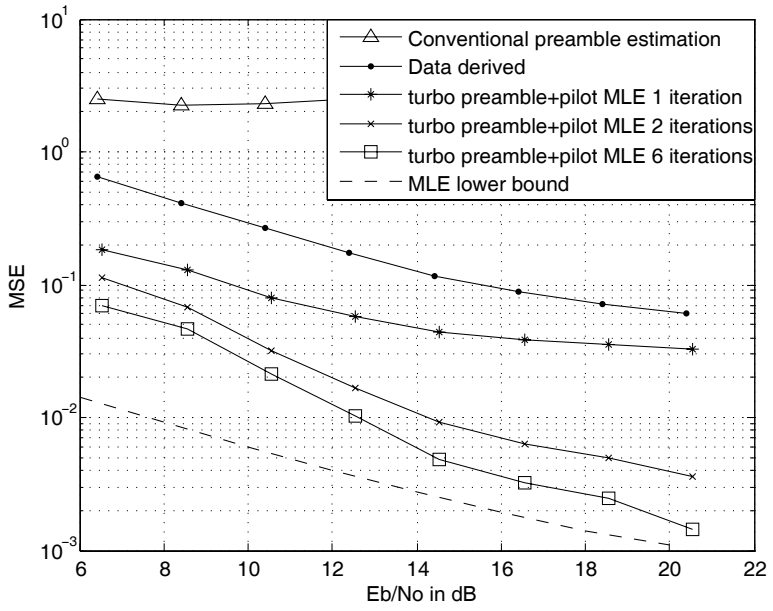

(b)

Fig. 4. Downlink performance between OFDM receiver with proposed iterative turbo channel estimation and OFDM receiver with conventional preamble channel estimation and data derived channel estimation (a) Frame Error Rate;(b) Mean Square Error.

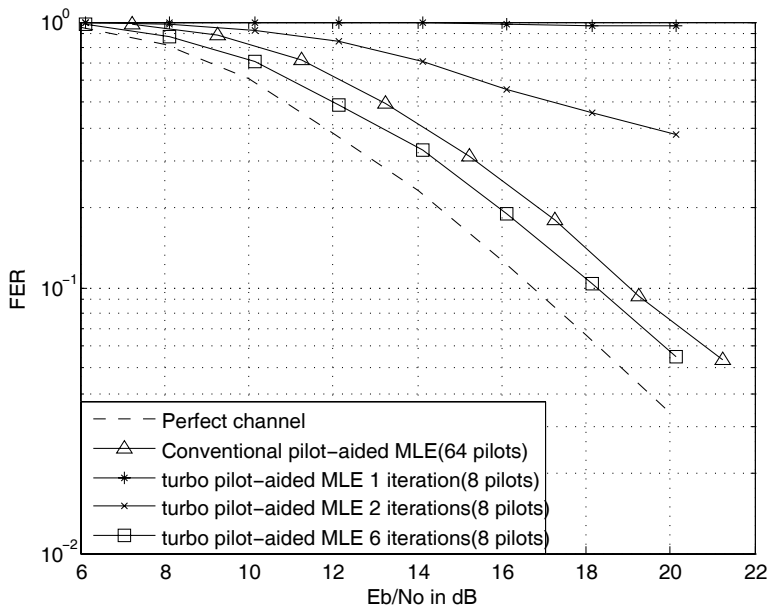

(a)

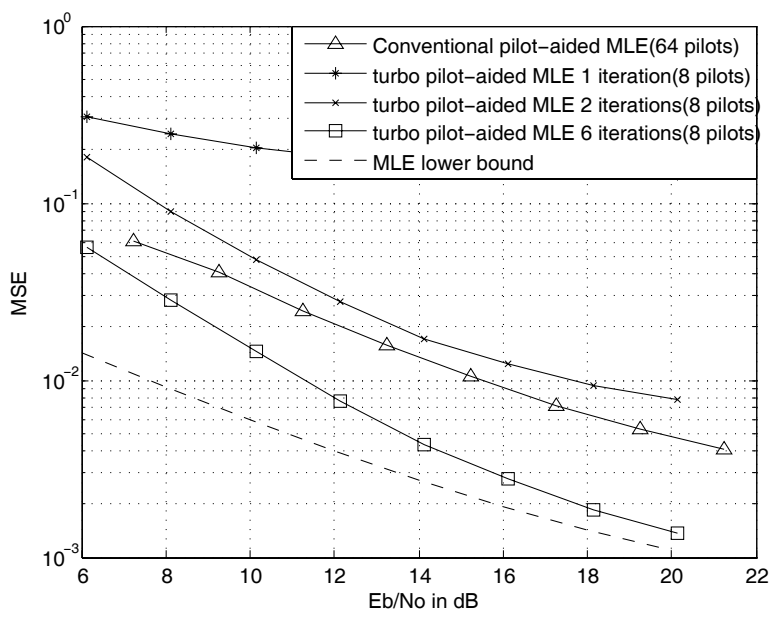

(b)

Fig. 5. Uplink performance between proposed OFDM receiver with iterative turbo channel estimation and OFDM receiver with conventional pilot-aided channel estimation (a) Frame Error Rate;(b) Mean Square Error.

the vehicular speed decreases. However, in all scenarios, the proposed OFDM receiver has a significant performance gain over other methods. It is also interesting to note that in this paper we assume ten data symbols per frame for simulations, while in IEEE802.16 [2], [3] standard the frame length can go up to 16 symbols. In that case, the channel variation can be considerable even at the vehicular speed of $60 \mathrm{kmh}$ and a more significant improvement can be achieved by our proposed receiver.

Finally, Fig. 8 shows the FER performance of the proposed iterative receiver with up to $4 \%$ uniform distributed residual CFO. It can be observed that the degradation ranges from a fraction of a dB for the $60 \mathrm{kmh}$ case to around $1 \mathrm{~dB}$ for the $333 \mathrm{kmh}$ case, compared to that of the CFO-free system. To address a more significant CFO, we can either employ a separate synchronization module to perform frequency error estimation and compensation, or mitigate the resultant ICI by advanced interference reduction algorithms [25]. Discussion on these approaches is beyond the scope of this paper.
To summarize, compared to OFDM receiver with existing channel estimation techniques, the proposed OFDM receiver with iterative turbo channel estimation can approach the MSE lower bounds. It approaches performance of perfect CSI even with a small number of preambles and pilots in a rapid dispersive fading channel, which makes it an efficient solution in terms of both SNR and throughput. Furthermore, the proposed iterative receiver is robust w.r.t residual CFO in practical OFDM systems.

\section{CONCLUSION}

In this paper, we address the problem of OFDM transmission in a rapid dispersive fading channel. In such a highly mobile environment, the wireless channel undergoes fast variations both in time and frequency. In order to track the fast varying channel, large number of pilot tones are usually inserted to the OFDM symbol for existing receivers, which incurs huge SNR and throughput loss. An iterative turbo channel estimation technique which makes use of preamble, 


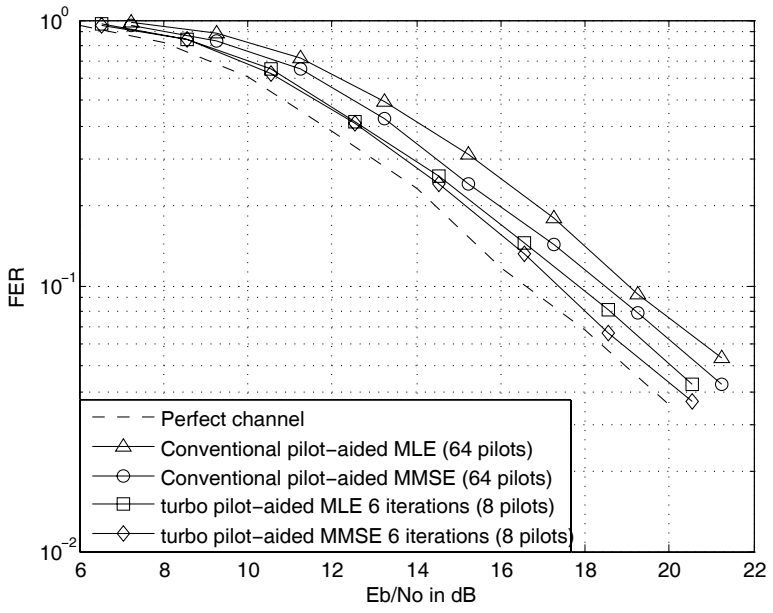

(a)

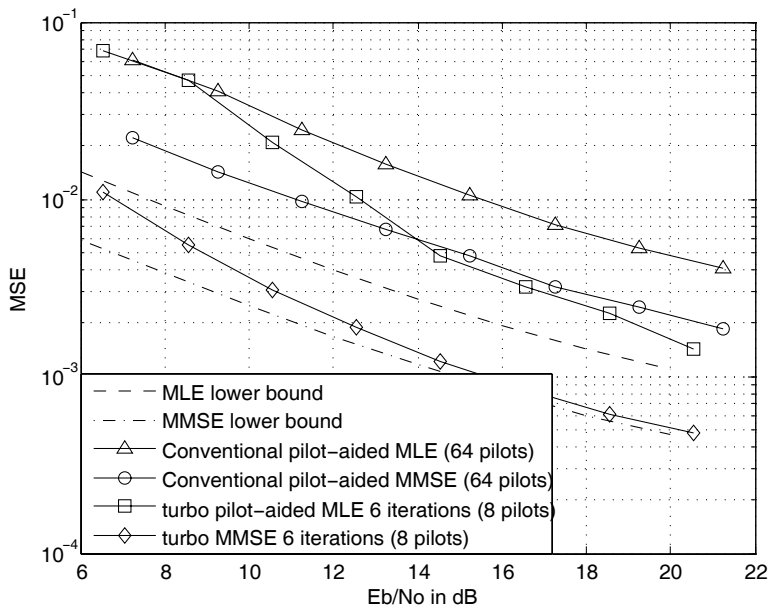

(b)

Fig. 6. Uplink performance between proposed OFDM receiver with iterative turbo MLE/MMSE channel estimation and OFDM receiver with conventional MLE/MMSE channel estimation (a) Frame Error Rate;(b) Mean Square Error.

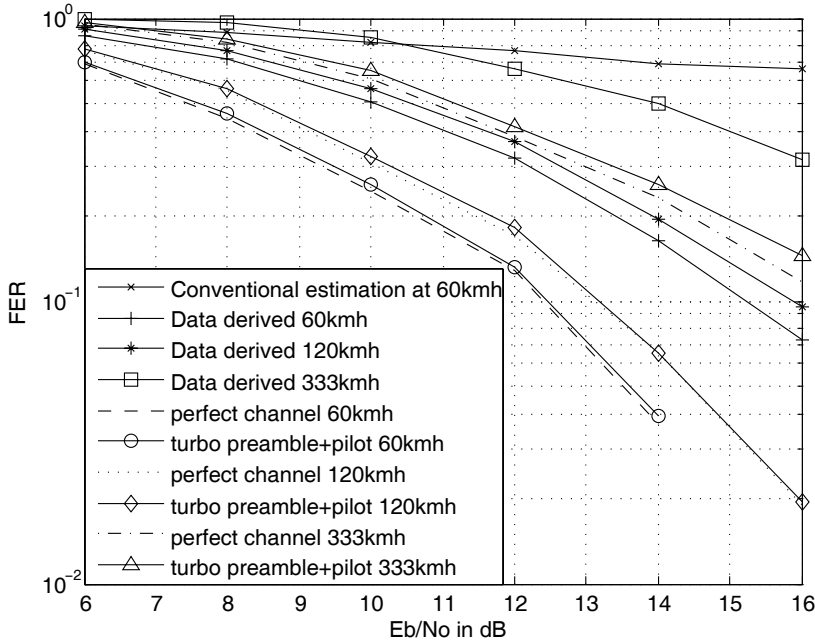

Fig. 7. Frame error rate performance between OFDM receiver with proposed iterative turbo channel estimation and OFDM receiver with conventional preamble channel estimation and data derived channel estimation [5] at different mobilities.

pilot and decoded soft data symbols in the channel estimation is proposed to improve the performance of channel estimation and maintain the system throughput at the same time. The channel estimation is conducted by three estimation stages, where a frequency-domain and time-domain combining strategy is proposed to combine the channel estimates from above signals in an efficient and low complexity manner. Numerical results and MSE analysis have shown that, compared to the OFDM receiver with existing channel estimation, the OFDM receiver with proposed iterative turbo channel estimation can approach the performance with nearly perfect CSI at various mobility scenarios. In addition to the improvement in both SNR and throughput benefits, the receiver is robust to frequency error and has low computational complexity which means it is possible to implement in hardware.

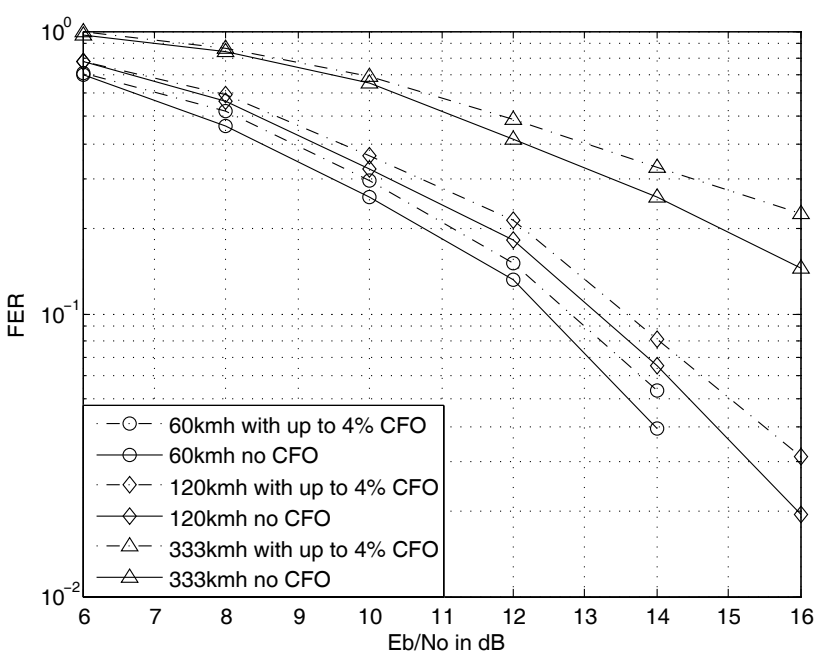

Fig. 8. Frame error rate performance of proposed OFDM receiver with up to $4 \%$ residue $\mathrm{CFO}$.

\section{ACKNOWLEDGMENT}

M. Zhao, Z. Shi and M. Reed are with National ICT Australia and affiliated with the Australian National University. National ICT Australia is funded through the Australian Governments Backing Australias Ability initiative and in part through the Australian Research Council.

\section{REFERENCES}

[1] Part 11: Wireless LAN Medium Access Control (MAC) and Physical Layer (PHY) Specification: High-Speed Physical Layer in the $5 \mathrm{GHz}$ Band, IEEE Std. 802.11a, 1999.

[2] Local and Metropolitan Area Networks-Part 16, Air Interface for Fixed Broadband Wireless Access systems, IEEE Std. 802.16a, 2003.

[3] Draft 12: Local and Metropolitan Area Networks-Part 16, Air Interface for Fixed Broadband Wireless Access systems, IEEE Std. 802.16e, 2005.

[4] Guidelines for evaluation of radio transmission technologies for IMT2000, ITU-T Std. M.1225, 1997.

[5] A. Dowler, A. Nix, and J. McGeehan, "Data-derived iterative channel estimation with channel tracking for a mobile fourth generation wide area OFDM system," in Proc. GLOBECOM, vol. 2, Dec. 2003, pp. 804808. 
[6] R. Negi and J. Cioffi, "Pilot tone selection for channel estimation in a mobile OFDM system," IEEE Trans. Consumer Electron., vol. 44, pp. 1122-1128, Aug. 1998.

[7] J. J. V. de Beek, O. Edfors, M. Sandell, S. K. Wilson, and P. O. Börjesson, "On channel estimation in OFDM systems," in Proc. IEEE VTC, Chicago, IL, July 1995, pp. 815-819.

[8] — "OFDM channel estimation by singular value decomposition," in Proc. IEEE VTC, Atlanta, GA, Apr. 1996, pp. 923-927.

[9] O. Edfors, M. Sandell, J. J. V. de Beek, S. K. Wilson, and P. O. Börjesson, "OFDM channel estimation by singular value decomposition," IEEE Trans. Commun., vol. 46, pp. 931-939, July 1998.

[10] V. Mignone and A. Morello, "CD3-OFDM, a novel demodulation scheme for fixed and mobile receivers," IEEE Trans. Commun., vol. 44, no. 12, pp. 1144-1151, Sept. 1996.

[11] Y. Li, L. J. Cimini, and N. R. Sollenberger, "Robust channel estimation for OFDM systems with rapid dispersive fading channels," IEEE Trans. Commun., vol. 46, no. 7, pp. 902-915, July 1998.

[12] A. Stamoulis, S. N. Diggavi, and N. Al-Dhahir, "Intercarrier interference in MIMO OFDM," IEEE Trans. Signal Processing, vol. 50, no. 10, pp. 2451-2464, Oct. 2002

[13] C. Y. Shin and E. J. Powers, "Double selective channel estimation for OFDM systems," in Proc. IEEE Asilomar, Pacific Grove, CA, Nov. 2005.

[14] P. Schniter, "Low-complexity estimation of double-selective channels," in Proc. IEEE SPAWC, Rome, Italy, June 2003, pp. 200-204.

[15] A. P. Kannu and P. Schniter, "Reduced complexity decision-directed pilot-aided tracking of double-selective channels," in Proc. CISS, Princeton, NJ, Mar. 2004, pp. 915-920.

[16] J. Hagenauer, "The turbo principle: Tutorial introduction and state of the art," in Proc. Int. Symp. Turbo Codes and Related Topics, Brest, France, Sept. 1997.

[17] Y. Li, N. Seshadri, and S. Ariyavisitakul, "Channel estimation for OFDM systems with transmitter diversity in mobile wireless channels," IEEE J. Select. Areas Commun., vol. 17, no. 3, pp. 461-471, Mar. 1999.

[18] Y. Li, "Simplied channel estimation for OFDM systems with multiple transmit antennas," IEEE Trans. Wireless Commun., vol. 1, no. 1, pp. 67-75, Jan. 2002.

[19] Y. Li, J. H. Winters, and N. R. Sollenberger, "MIMO-OFDM for wireless communications: Signal detection with enhanced channel estimation," IEEE Trans. Commun., vol. 50, no. 9, pp. 1471-1477, Sept. 2002.

[20] B. Song, W. Zhang, and L. Gui, "Iterative joint channel estimation and signal detection in MIMO OFDM system," in Proc. IEEE WCNM, Sept. 2005, pp. 39-43.

[21] B. Lu, X. Wang, and Y. Li, "Iterative receivers for space-time block coded OFDM systems in dispersive fading channels," IEEE Trans. Wireless Commun., vol. 1, pp. 213-225, Apr. 2002.

[22] Y. Sun, Z. Xiong, and X. Wang, "EM-based iterative receiver design with carrier frequency offset estimation for MIMO OFDM systems," IEEE Trans. Commun., vol. 53, no. 4, pp. 581-586, Apr. 2005.

[23] J. Moon, H. Jin, T. Jeon, and S. K. Lee, "Channel estimation for MIMOOFDM systems employing spatial multiplexing," in Proc. IEEE VTCFall, Sept. 2004, pp. 3649-3654.

[24] S. Y. Park, Y. G. Kim, and C. G. Kang, "Iterative receiver for joint detection and channel estimation in OFDM systems under mobile radio channels," IEEE Trans. Veh. Technol., vol. 53, pp. 450-460, Mar. 2004.

[25] S. Tomasin, A. Gorokhov, H. Yang, and J. P. Linnartz, "Iterative interference cancellation and channel estimation for mobile OFDM,' IEEE Trans. Wireless Commun., vol. 4, pp. 238-245, Jan. 2005.

[26] W. C. Jakes, Microwave Mobile Communications. IEEE Press, 1994.

[27] W. G. Jeon, K. H. Chang, and Y. S. Cho, "An equalization technique for orthogonal frequency-division multiplexing system in time-variant multipath channel," IEEE Trans. Commun., vol. 47, pp. 27-32, Jan. 1999.
[28] M. Zhao, Z. Shi, and M. C. Reed, "Iterative turbo channel estimation for OFDM system over rapid dispersive fading channel," in Proc. IEEE ICC, Glasgow, Scotland, June 2007.

[29] S. M. Kay, Fundamentals of Statistical Signal Processing: Estimation Theory. Englewood Cliffs, NJ: Prentice-Hall, 1993.

[30] M. Morelli and U. Mengali, "A comparison of pilot-aided channel estimation methods for OFDM systems," IEEE Trans. Signal Processing, vol. 49, no. 12, pp. 3065-3073, Dec. 2001.

[31] H. Liu and G. Q. Li, OFDM-Based Broadband Wireless Networks: Design and Optimization. Wiley, 2005.

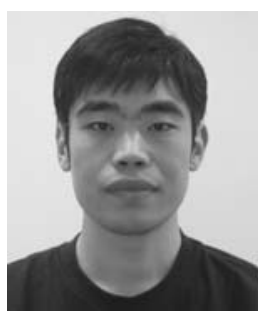

Ming Zhao (S'03) received the B.Eng. and M.Eng. degrees from the National University of Singapore (NUS), Singapore, in 2003 and 2005, respectively, both in electrical engineering. Since 2005, he has been with National ICT Australia (NICTA), and currently is pursuing Ph.D degree in Research School of Information Science and Engineering (RSISE), Australian National University (ANU). His area of research includes turbo principle, iterative wireless signal processing, MIMO-OFDM system, robust image and video coding. Mr. Zhao is a student member of IEEE Communications Society since 2003.

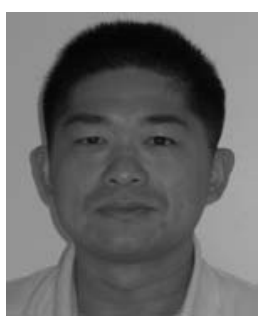

Zhenning Shi received his BS degree in Electronics Engineering from Tsinghua University, China, in 1998, and his $\mathrm{PhD}$ degree in Electrical and Computer Engineering from the University of Utah, US, in 2003. His doctoral research is on analysis and algorithm development for iterative multiuser receivers. From 2002 to 2004, he worked as a post-doctoral fellow at the Electrical and Computer Engineering Department, University of Utah, in collaboration with L-3 Communications on a project to develop low-complexity multiuser detection methods based on Monte-Carlo Markov Chain (MCMC). He is currently a research staff member at National ICT Australia, and is an adjunct research fellow at Australian National University (ANU). His recent work has been focused on signal processing techniques for OFDM-based systems.

Zhenning Shi's primary research interests include the Turbo receiver design, multiuser detection, synchronization, channel estimation and equalization in time-frequency selective channels, CDMA, MIMO, and OFDMA systems. $\mathrm{He}$ has published 40 journal and conference papers, and is listed as an author on four patent applications.

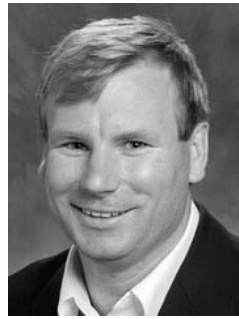

Mark C. Reed has worked in industry and research positions for the last 17 years with positions in the U.S.A., Switzerland, and Australia. He pioneered the area of iterative receiver design as part of his doctoral studies and was part of a team that designed and developed a world first Satellite-UMTS Modem for the European Space Agency. He also completed further work on $3 \mathrm{G}$ Basestation design as technical lead in the highly successful European Commission project, ASILUM, which investigated and validated advanced signal processing schemes for link improvement in UMTS. Since April 2003 Dr Reed is employed as a Principle Researcher at the National ICT Australia (NICTA), and is an Adjunct Assoc. Professor at the Australian National University, Canberra, Australia, where he is involved in research, education, and commercialization, in wireless signal processing. He has over 50 international journal and conference papers, he was an Associate Editor for the IEEE Transactions on Vehicular Technology (2005-2007), and has been listed as inventor on seven patent applications. 